\title{
Correlation between current and cross-sectional area of parallel fixed-movable dual electrodes in ESC
}

\author{
${ }^{*}$ Li-ting Zhao ${ }^{1,2}$, Yun-long Xiong ${ }^{1,2}$, Rui Chen ${ }^{1,2}$, Zeng-rui Wang ${ }^{1,2}$, An-guo Wang ${ }^{3}$ \\ 1. State Key Laboratory of Light Alloy Casting Technology for High-end Equipment, Shenyang 110022, China \\ 2. Shenyang Research Institute of Foundry Co., Ltd., Shenyang 110022, China \\ 3. School of Materials Science and Engineering, Shenyang Aerospace University, Shenyang 110136, China
}

\begin{abstract}
Electroslag casting with parallel fixed-movable dual electrodes is a new method for achieving better quality of castings in complex mold cavities. In this work, a mathematical model, $y=k x^{2}+(k+1) x$, was established to describe the ideal correlation between the current ratio $(y)$ and the cross-sectional area ratio $(x)$ of the dual electrodes, where $k$ is the filling ratio. Investigation was conducted on the electroslag casting process with dual electrodes of various cross-sectional areas, but at a constant $k$ value. The experimental results indicated that the ideal correlation was obtained at the stable casting stage, and the fitting results were consistent with the experimental results at certain $k$ values. The experimental findings show that better castings can be obtained when the current ratio is greater than 1.536 and the cross-sectional area ratio is greater than 0.5 .
\end{abstract}

Key words: electroslag casting; fixed-movable dual electrode; current ratio; cross-sectional area ratio CLC numbers: TG249.3 Document code: A

Article ID: 1672-6421(2020)03-245-05

E lectroslag casting (ESC) has been widely used for refining and solidification processes ${ }^{[1-4]}$. Traditional ESC methods, including electroslag one-step casting (ESOSC), electroslag step-by-step casting (ESBSC), electroslag pouring (ESP) and centrifugal electroslag casting (CESC), have been applied in producing specialshaped castings, such as rotary bodies, crankshafts, and turbine blades ${ }^{[5-14]}$. In ESOSC, to obtain a qualified part, the mold cavity needs to be enlarged to increase the casting allowances. Then, the excessive material must be removed from the part by machining after casting. Thus, ESOSC is both time consuming and expensive. Although ESBSC can partly solve the related casting issues, this method cannot guarantee the mechanical properties and chemical composition of the joined parts. ESP and CESC cannot be widely used because these methods require multi-tooling cooperative operations.

The schematic drawing of conventional ESC setup and different cavities is shown in Fig. 1. To solve the problem of manufacturing castings with complex mold cavities by ESC, a new ESC method using parallel fixed-movable dual electrodes was proposed ${ }^{[15]}$. Figure 2 shows that, in contrast to traditional ESC, this new method uses two

\section{*Li-ting Zhao}

Male, born in 1992, engineer. His research interests mainly focus on material forming technology.

E-mail: zlt9224@qq.com

Received: 2019-08-28; Accepted: 2020-03-26 types of electrodes: a fixed consumable electrode and a movable consumable electrode. The shape of the fixed consumable electrode is roughly the same as that of the mold but smaller. This electrode is fixed inside the mold and remains in this position during the ESC process. The fixed electrode can provide partial liquid in the metal pool, and the rest of the mold could be filled by the movable electrode. The combination of two electrodes increases the mold-filling capacity by decreasing the transfer distance and liquid volume in the metal pool. Therefore, castings with better quality can be achieved.

As a new method of ESC, parallel fixed-movable dual-electrode ESC needs to be further studied. In this work, a mathematical model was established to study the correlation between the current and the crosssectional area of the dual electrodes, and suitable ratios of the current and cross-sectional area were determined for high quality castings.

\section{Experimental procedure}

Figure 3 shows a schematic of a parallel fixed-movable dual-electrode electroslag remelting (ESR) furnace. The setup is simplified for plate casting for the current study. A fixed consumable electrode is placed and secured inside the mold, and a movable consumable electrode is fixed on the arm of the furnace. The technical parameters of the ESR furnace are as follows:

(1) A $3 \mathrm{t}$ ingot can be cast in a water-cooled mold with 


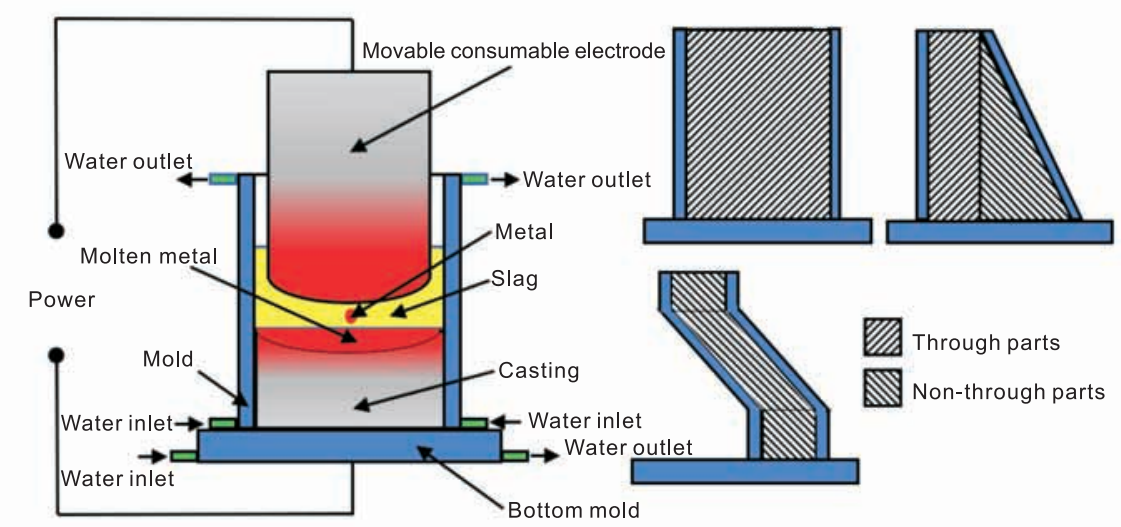

Fig. 1: (a) Schematic drawing of conventional ESC setup; (b) different cavities

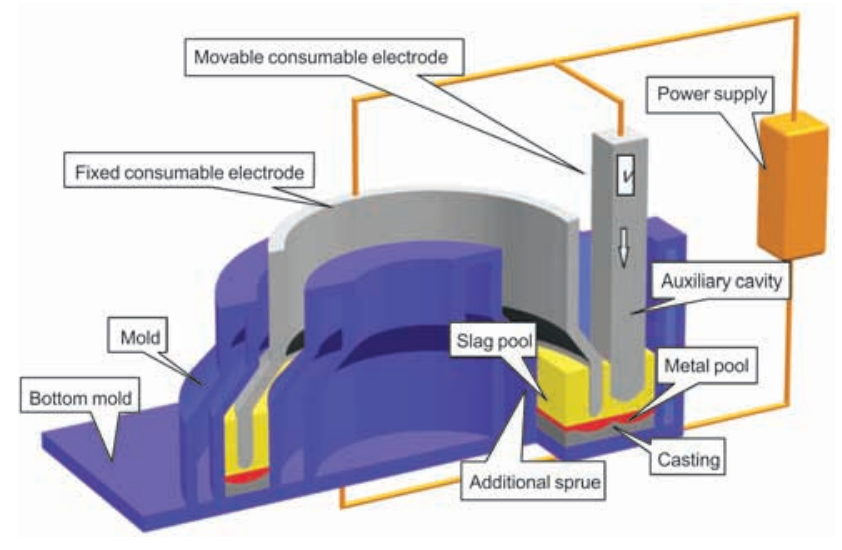

Fig. 2: Schematic drawing of the parallel fixed-movable dual-electrode ESC setup

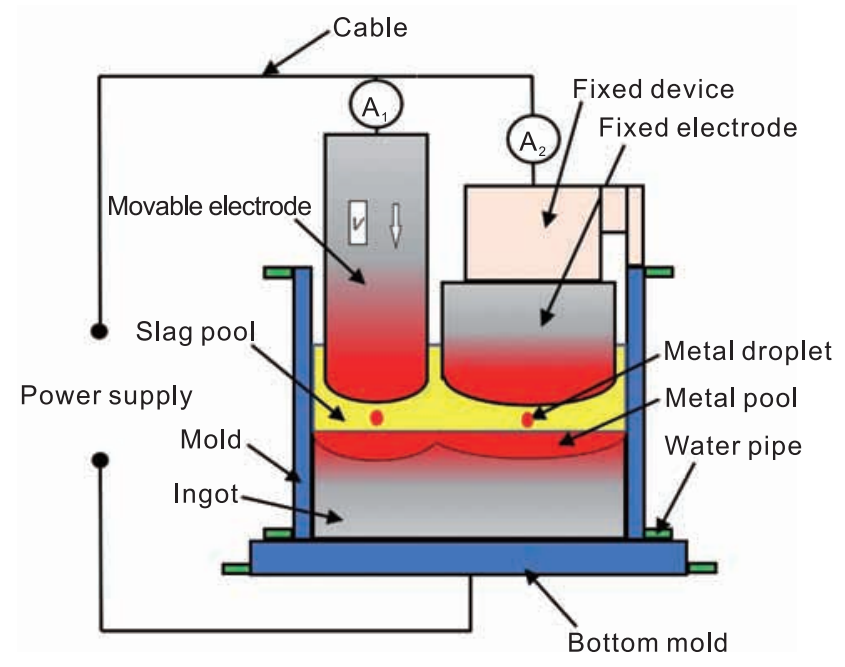

Fig. 3: Schematic drawing of the parallel fixed-movable dual-electrode ESR furnace

a $500 \mathrm{~mm}$ bottom diameter and a $600 \mathrm{~mm}$ top diameter.

(2) A $280 \mathrm{kVA}$ AC supply is available to produce ESR ingots with a high-voltage terminal of $380 \mathrm{~V}$, a low-voltage terminal ranging from $28 \mathrm{~V}$ to $80 \mathrm{~V}$, and a maximum current of $10,000 \mathrm{~A}$.

(3) The electroslag furnace is automatically controlled, that is, the movable consumable electrode can be driven up and down at a certain speed based on predetermined electrical parameters.
The current passing through the electrodes was measured with a rectified digital multimeter at the rate of once per minute by an induction coil placed around the cable.

The dimensions of the experimental electrodes and the mold are shown in Table 1.

Table 1: Dimensions of dual electrodes and mold

\begin{tabular}{cccc} 
Experiment & Mold $(\mathrm{mm})$ & $\begin{array}{c}\text { Fixed } \\
\text { consumable } \\
\text { electrode }(\mathrm{mm})\end{array}$ & $\begin{array}{c}\text { Movable } \\
\text { consumable } \\
\text { electrode }(\mathrm{mm})\end{array}$ \\
\hline No. 1 & $400 \times 70 \times 500$ & $260 \times 50 \times 400$ & $100 \times 50 \times 3,000$ \\
No. 2 & $400 \times 70 \times 500$ & $240 \times 50 \times 400$ & $120 \times 50 \times 3,000$ \\
No. 3 & $400 \times 70 \times 500$ & $180 \times 50 \times 400$ & $180 \times 50 \times 3,000$ \\
No. 4 & $400 \times 70 \times 500$ & $180 \times 50 \times 400$ & $180 \times 50 \times 3,000$ \\
No. 5 & $400 \times 70 \times 500$ & $120 \times 50 \times 400$ & $240 \times 50 \times 3,000$ \\
No. 6 & $400 \times 70 \times 500$ & $100 \times 50 \times 400$ & $260 \times 50 \times 3,000$ \\
\hline
\end{tabular}

Q235 bearing steel containing $0.14 \%-0.22 \% \mathrm{C}, 0.30 \%-$ $0.65 \% \mathrm{Mn}, 0.2 \%-0.3 \% \mathrm{Si}$, and $1.4 \%-1.5 \% \mathrm{Cr}$ was used in this study. The slag used was $30 \% \mathrm{Al}_{2} \mathrm{O}_{3}+70 \% \mathrm{CaF}_{2}$. The casting process started with $20 \mathrm{~kg}$ of solid slag. The arc striking agent was composed of $50 \% \mathrm{CaF}_{2}$ and $50 \% \mathrm{TiO}_{2}$. The arc voltage was $34 \mathrm{~V}$, the current was 3,000 A, and the normal melting voltage and current were $60 \mathrm{~V}$ and 7,000 A, respectively. The filling ratio was 0.357 .

After the fixed consumable electrode completely melted, the movable consumable electrode continued feeding until the mold cavity was filled.

\section{Results and discussion}

\subsection{Mathematical model}

The model assumes that the fixed consumable electrode is fixed on the mold and the movable consumable electrode keeps feeding to maintain the slag liquid level and keeps a constant distance between poles. Under the skin effect of 
the current and the gravity effect of the droplets, the end of the consumable electrode and the molten pool are parabolic in the ESC process. The model assumes that the end of the consumable electrode and the molten pool stay level.

The following definitions are made for the movable consumable electrode: the electrode length is $L_{1}$, the crosssectional area is $S_{1}$, the melting distance per unit time is $\Delta L_{1}$, the melting current is $I_{1}$, the resistance is $R_{1}$, and the current density is $J_{1}$. Then, the following definitions are made for the fixed consumable electrode: the electrode length is $L_{2}$, the cross-sectional area is $S_{2}$. The melting distance per unit time is $\Delta L_{2}$, the melting current is $I_{2}$, the resistance is $R_{2}$, and the current density is $J_{2}$. The electrode length change during ESC is shown in Fig. 4.

According to conservation of mass, the total molten steel volume melted by the movable consumable electrode and fixed consumable electrode is equal to the solidified volume of molten steel in the mold:

$$
\begin{gathered}
\Delta L_{1} \cdot S_{1}+\Delta L_{2} \cdot S_{2}=\Delta L_{2} \cdot \mathrm{S} \\
\frac{\Delta L_{1}}{\Delta L_{2}}=\frac{S-S_{2}}{S_{1}}
\end{gathered}
$$

Thus:

$$
I_{1} \cdot \rho \frac{L_{1}}{S_{1}}=I_{2} \cdot \rho \frac{L_{2}}{S_{2}}
$$

Substituting Eq. (2) into (3), then:

$$
\frac{I_{1}}{I_{2}}=\frac{S_{1}^{2}}{S_{2}\left(S-S_{2}\right)}
$$

By defining the filling ratio of the electrodes as $k$, the following expression can be obtained:

$$
\left(S_{1}+S_{2}\right) \cdot(k+1)=S
$$

Substituting Eq. (5) into (4) yields the following expression:

$$
\begin{gathered}
\frac{I_{1}}{I_{2}}=k \cdot\left(\frac{S_{1}}{S_{2}}\right)^{2}+(k+1) \cdot \frac{S_{1}}{S_{2}} \\
\text { Let } y=\frac{I_{1}}{I_{2}} \text { and } x=\frac{S_{1}}{S_{2}} \text {, then } y=k x^{2}+(k+1) x \\
\text { Let } z=\frac{J_{1}}{J_{2}} \text { and } x=\frac{S_{1}}{S_{2}} \text {, then } z=k x+k+1
\end{gathered}
$$

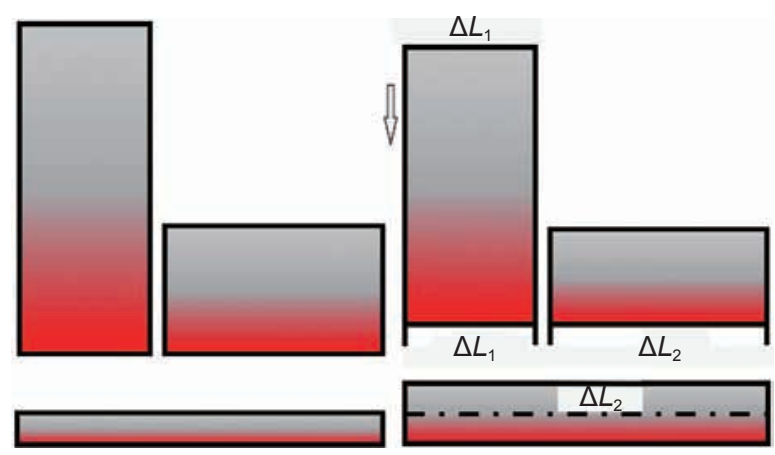

Fig. 4: Drawing of casting process

\subsection{Current correlations between two electrodes}

The current change in the dual electrodes during the experiment is shown in Figs. 5a-5f. The process could be divided into three stages: electrode ignition, current rising and casting stabilization.

In the ignition stage, the movable consumable electrode was initially charged, beginning the electroslag casting process. Then, the slag material starts melting, together with the fixed consumable electrode being charged and melted. In the current rising stage, increasing the current will improve the electrode volatility and the melting rate. In the stabilization stage, the (a)

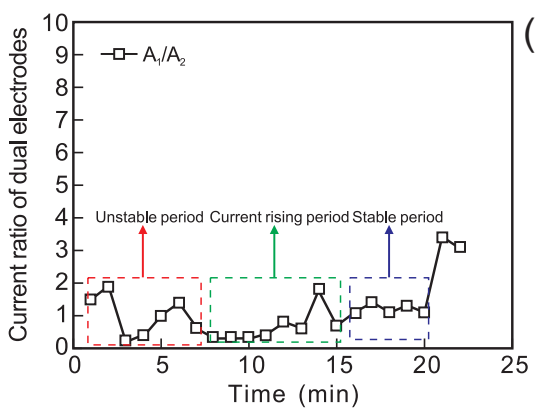

(d)

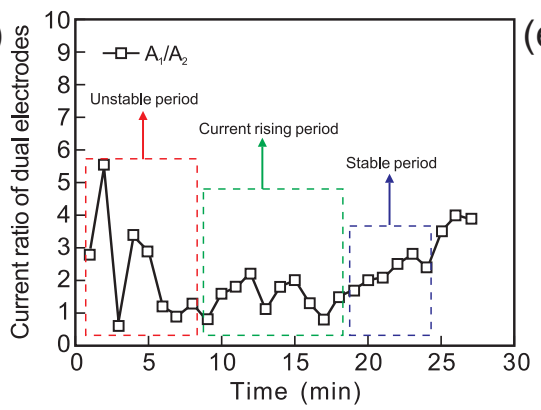

(b)

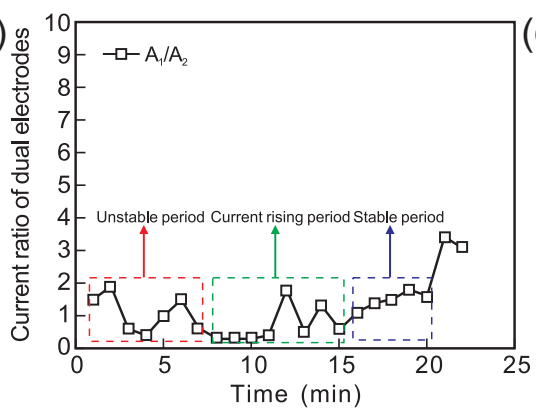

e)

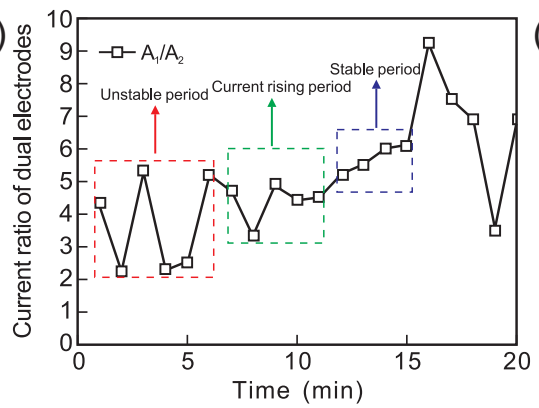

c)

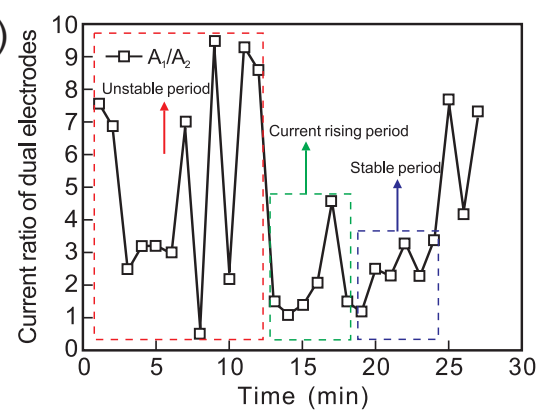

(f)

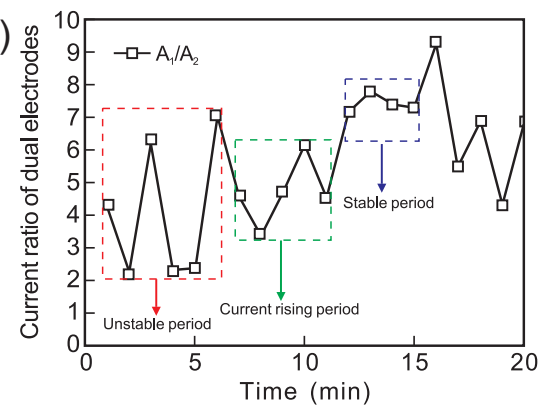



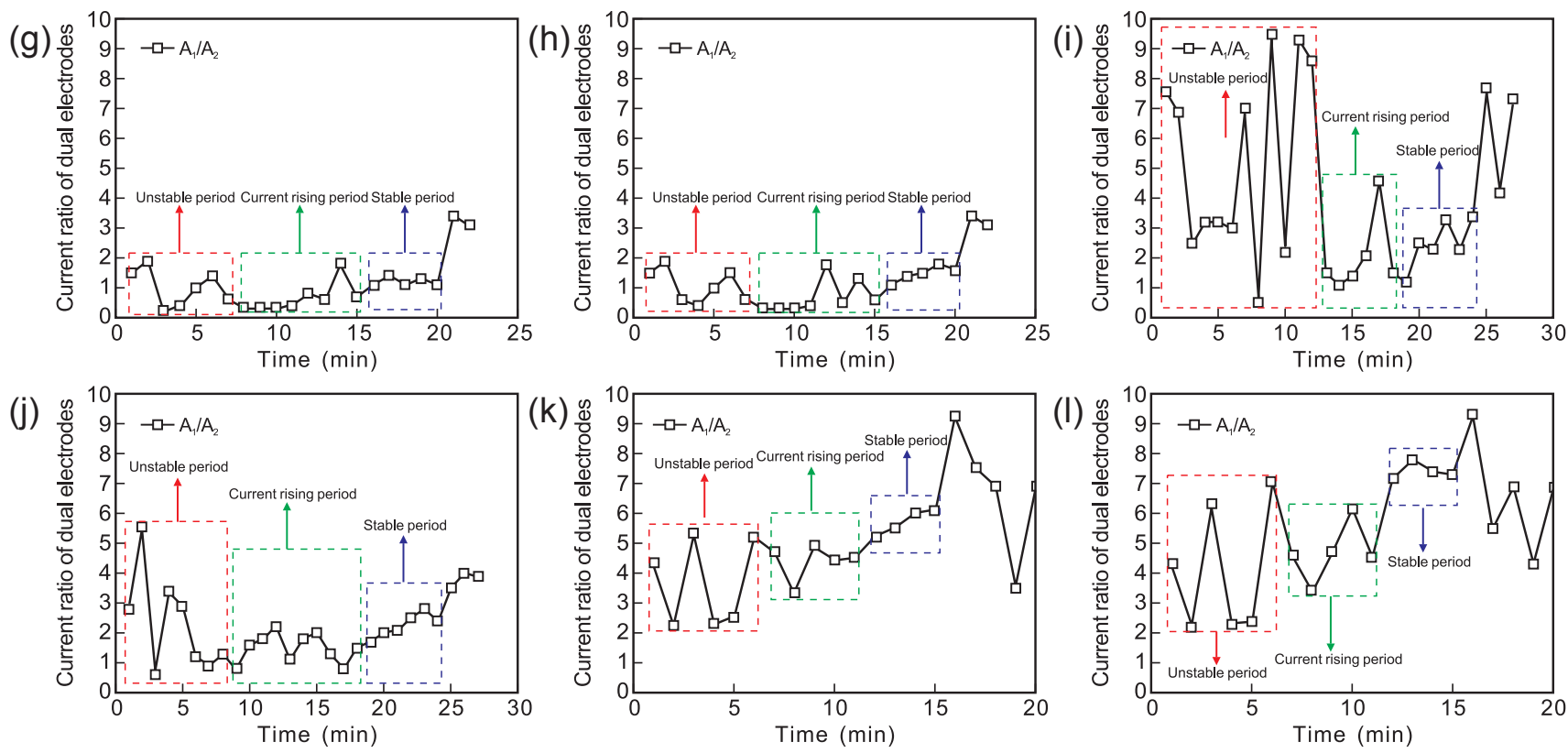

Fig. 5: Current changes in the electrodes (a-f) and current ratio changes in the dual electrodes (g-l)

sum of current through both electrodes tends to the rated current. Generally, fluctuations occur above or below the rated current.

The current ratio changes in the dual electrodes in the three stages are illustrated in Figs. 5g-51. In both the ignition and the current rising stages, the current ratio fluctuates within a wide range.

Figure 6 shows that in the stable casting stage, there is a quadratic relationship between the current ratio and the crosssectional area ratio of the dual electrodes. Let $y=a x^{2}+b x+c$, the values of $a$ and $b$ are obtained by quadratic function fitting through the above data of six experiments, and $a=0.3564$, $b=1.3578, c=0$. The filling ratio set in the experiment is 0.357 . The experimental fitting results are basically in agreement with the calculations from Eq. (7).

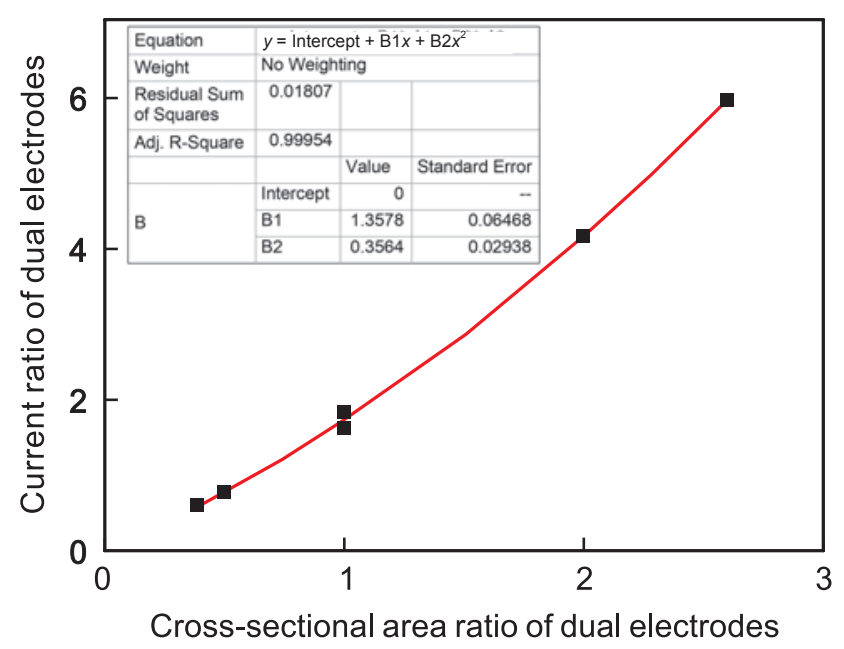

Fig. 6: Relationship between the current ratio and the cross-sectional area ratio of dual electrodes

\subsection{Determination of optimum casting parameters}

Figure 7 shows the appearance of the ingots Nos. 2, 3, 4 and 5. The surface quality is better in ingots Nos. 3, 4 and 5. Slag ditch was found in ingot No. 2, indicating worse surface quality. Table 2 shows the current density ratio of the dual electrodes calculated by Eq. (8). In this method, the dual electrodes adopt a free distribution mechanism, and the rated total current is constant. When the filling ratio is kept constant, the larger the cross-sectional area of the fixed consumable electrode, the more molten steel needed to supply the mold cavity per unit time; thus, the melting speed of the movable consumable electrode must increase. When the crosssectional area ratio is 0.5 , the current density ratio is 1.536 . The actual melting rate of the movable consumable electrode is too low to reach the minimum value which can ensure the perfect solidification, so slag ditch is formed in the middle of the ingot near the movable consumable electrode side. When the current density ratio of the dual electrodes is greater than 1.536 , the ingot with better quality can be achieved.

\section{Conclusion}

The present study using a new ESC method with parallel fixedmovable dual electrodes to obtain simplified plate castings. Experimental results reveal that this casting process principle is feasible and promising. The analysis of the current and the cross-sectional area between the dual electrodes and the evaluation of the melted ingots appearance provide theoretical guidance to achieve better quality castings in complex mold cavities. The ideal correlation between the current ratio $(y)$ and the cross-sectional area ratio $(x)$ of the dual electrodes could be mathematically described as $y=k x^{2}+(k+1) x$, where $k$ is the filling ratio. This ideal correlation was identified during 


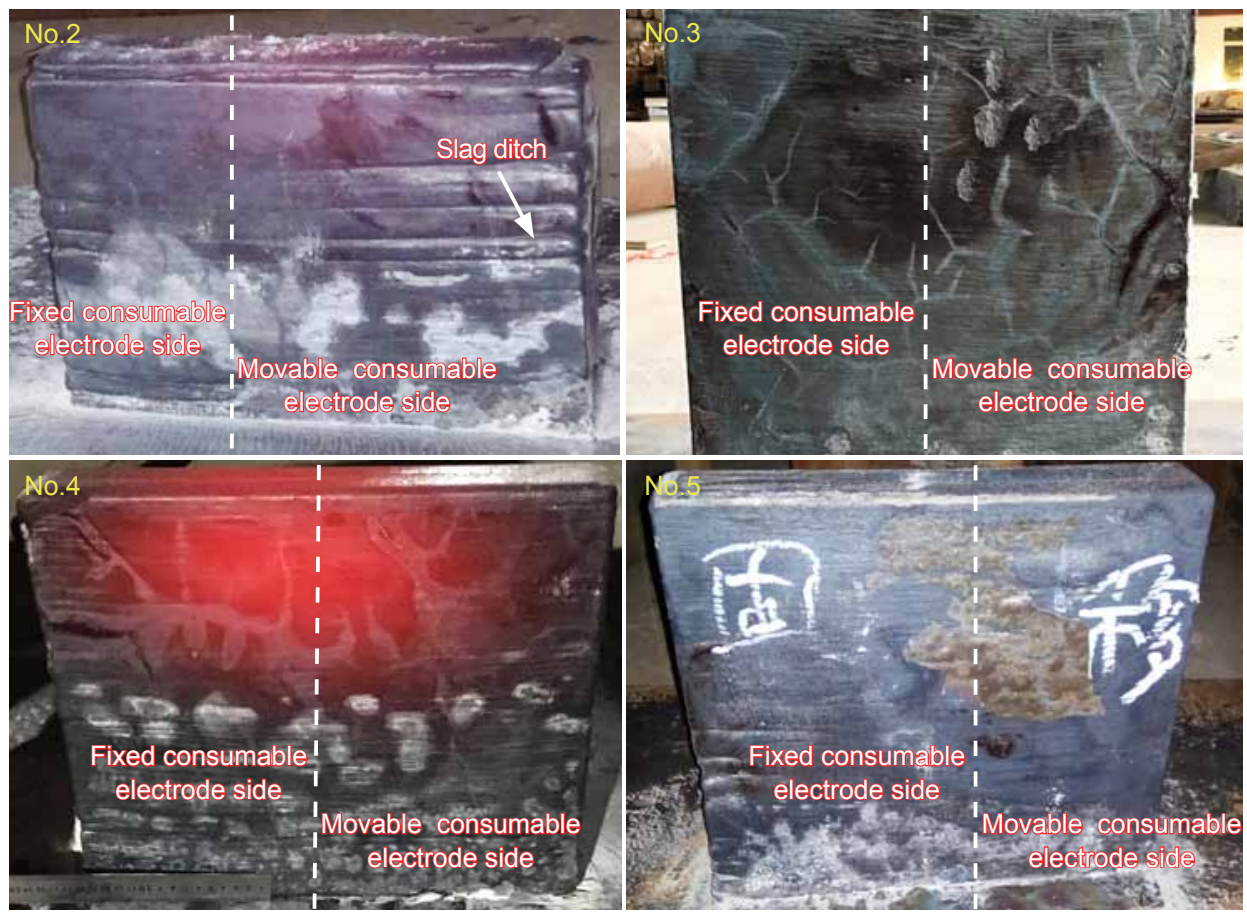

Fig. 7: Appearance of ingots

Table 2: Current density ratio of dual electrodes

\begin{tabular}{ccc} 
Experiment & Calculated ratio & Actual ratio \\
No. 2 & $1+1.5 k$ & 1.536 \\
No. 3 & $1+2 k$ & 1.714 \\
No. 4 & $1+2 k$ & 1.714 \\
No. 5 & $1+3 k$ & 2.071 \\
\hline
\end{tabular}

the stable casting stage, where good agreement was found between the fitting and experimental results at certain $\mathrm{k}$ values. The experimental results suggested that better quality castings could be achieved when the current ratio was greater than 1.536 and the cross-sectional area ratio was greater than 0.5 .

\section{References}

[1] Paton B E, Medovar B I, Marinski G S, et al. Contemporary electroslag crucible melting and casting, and its future outlook. Electroslag Technology, Springer, New York, NY, 1991: 3-9.

[2] Parsons R C. Electroslag melting and casting process. US Patent No. US3511303, 1970.

[3] Leonidovich A V, Ippolitovi B A, Vasilievich C L, et al. Device for the electroslag casting of ingots. US Patent No. 3924672 A, 1975.

[4] Li Z B. Development of electroslag metallurgy and casting in China. China Foundry, 2004, 1(1):7-16.

[5] Wang Z, Xu J, Bao G, et al. Fabrication of high-power diesel engine crankshafts by electro-slag casting process. Journal of Materials Processing Technology, 2007, 182(1-3): 588-592.
[6] Shi X, Wan Z M, Liu J W, et al. One step electroslag melt-casting process for integral crankshaft and equipment. CN Patent No. 1006531 B, 1990. (In Chinese)

[7] Bu W L, Jiang J B. A study on the inherent qualities of electroslag meltings cast crank-shaft by one step. Chinese Internal Combustion Engine Engineering, 1994 (2): 12. (In Chinese)

[8] Liu X, Hu X, Geng C, et al. Study on electroslag pouring process of nonuniform slab. Journal of Iron and Steel Research, 1996 (3): 10-14. (In Chinese)

[9] Zhang Z G. Crankshaft blank produced with electroslag step by step casting. Hot Working Technology, 2005 (3): 03. (In Chinese)

[10] Zherebtsov S N, Fat'Yanov S V. Centrifugal electroslag casting of annular flange blanks. Chemical \& Petroleum Engineering, 2005, 41 (5-6): 348-350.

[11] Wang Z, Xu J, Bao G, et al. Fabrication of high-power diesel engine crankshafts by electro-slag casting process. Journal of Materials Processing Technology, 2007, 182 (1): 588-592. (In Chinese)

[12] Chen R, Li B D, Wang H F, et al. Aluminium alloy crystallizer for electroslag fusion casting of small guide blade: CN Patent No. 1974073 A, 2007. (In Chinese)

[13] Jiang $Y$ F, Zhang $Y$ L, Zhang J D, et al. Electroslag smelting casting method of fixed blades of water turbine. CN Patent No. 2012101607306 B, 2012. (In Chinese)

[14] Zherebtsov S N, Mikhailets S N, Zabegailo I V, et al. Use of centrifugal electroslag casting for producing cold-resistant steel grade ring blanks of flange objects. Chemical and Petroleum Engineering, 2017, 53 (5-6): 347-352.

[15] Zhao L T, Li B D, Xiong Y L, et al. Study on dynamic characteristics of the filling method of fixed consumable electrode. Foundry, 2017 (7): 727-730. (In Chinese) 